INTERNATIONAL BULLETIN OF BACTERIOLOGICAL NOMENCLATURE AND TAXONOMY

Volume 11, No. 4 October 15, 1961

pp. 133-159

\title{
ON EXTERNAL FEATURES IN THE TAXONOMY OF ACTINOMYCETES
}

N.A. Krasil'nikov, N.I. Nikitina, and A.I. Korenjako

\author{
Institute of Microbiology \\ Academy of Sciences \\ Moscow, USSR
}

SUMMARY: The usefulness of several characters in the differentiation of taxa included in the Actinomycetales is reviewed. Groups (taxa) are defined in each case not by a single character but by a combination of several. The colourless actinomycetes with white aerial mycelium include one group with straight sporophores and one with spiral. In green, violet and blue groups the solubility of pigments produced and the nature of the fructification are important. If the groups are established according to external morphological and cultural characters, then the species included in the groups are differentiated according to the physiological, biochemical and antimicrobial characters and the specificity of mutual antagonisms. Within the same group the species differ in the complex of characters. In the globisporus group 8 species are recognized, 7 species and 2 subspecies in the violet group, in the bluish white group, 2 species, in the brown-green group 2 species and in the fluorescent group 3 species.

The problems of taxonomy, the extablishment and identification of species of actinomycetes are currently regarded as most important and urgen in antibiotic science.

We have repeatedly noted that the confusion existing in this field greatly impedes the work on finding and studying antibiotics. This circumstance has caused the Inte rnational Taxonomy Committee to set up a species Subcommittee on Taxonomy of Actinomycetes. 
At the first sitting of this Subcommittee, which was held in Stockholm in August 1958, it was decided to carry out an international study of a specially selected collection of 25 actinomycetes. Thirty-two laboratories in various countries -Germany, Italy, Japan, USA, USSR and others-have taken part in the processing of these cultures.

The cultures were studied according to an established scheme and program by identical methods using identical nutrient media. The study continued for about two years. The results of the study were collected by the Subcommittee, (Secretary Dr. Küster and others) and duly processed. A brief summary of this processing of the experimental material has been circulated among the participants.

The results obtained are far from uniform. The same features in the same strains are represented differently. Even morphological indications such as the shape and the structure of sporophores and spores yield widely divergent data. For instance, of 87 analyses of strain No.1 (made in 32 laboratories), 17 show straight sporophores and 70 spiral ones; of 88 analyses of strain No.2, 77 show straight sporophores and 11 spiral. Similar data were obtained for the remaining cultures (Table 1 ).

As to spores, the results are more definite and in better agreement. Here too, however, some divergency is observed. For instance, in 32 analyses of strain No.l the spore membrane is given as smooth, while in one case it is prickly; in 3 analyses of strain No.12 the spore surface is defined as smooth, in 28 analyses as spiny; strain No. 21 in three cases had smooth spores, in 27 cases spiny, and in one case hairy. In all, such discrepancies in respect to spores amount to 11 out of 32 analyses.

There is a divergence of opinion and results in establishing the mode of branching of mycelium in the tested 25 cultures of actinomycetes. The same strains exhibit either only monopodial branching or monopodial and verticillate, the verticils being both primary and secondary. For instance, 8 analyses of strain No.l yield primary verticils with spiral sporophores, while one analysis gives secondary verticils and straight sporophores. Similar results were obtained for strains Nos.8,21. Strains 2,3,6, 7, 10 in the majority of cases exhibit monopodial structure of sporophores, though there are individual cases of verticillate structure (Table 2). 
Page 135

B ACTERIOLOGICAL NOMENCLATURE

AND TAXONOMY

Table 1. Structure of sporophores and spores according to data from various laboratories of the world. Number of strains with a given character.

\begin{tabular}{|c|c|c|c|c|c|c|}
\hline \multirow{2}{*}{\multicolumn{2}{|c|}{$\begin{array}{l}\text { Actino- } \\
\text { mycetes }\end{array}$}} & \multicolumn{2}{|c|}{ Sporophores } & \multicolumn{3}{|c|}{ Spores } \\
\hline & & Spiral & Nonspiral & Smooth & Spiny & Hairy \\
\hline \multicolumn{2}{|c|}{ Strain 1} & 70 & 17 & 32 & 1 & 0 \\
\hline 11 & 2 & 11 & 77 & 31 & 1 & 0 \\
\hline$"$ & 3 & 7 & 79 & 33 & 0 & 0 \\
\hline$" 1$ & 4 & 89 & 9 & 32 & 0 & 0 \\
\hline$"$ & 5 & 82 & 8 & 31 & 1 & 0 \\
\hline$"$ & 6 & 12 & 81 & 34 & 0 & 0 \\
\hline$" 1$ & 7 & 89 & 25 & 26 & 0 & 1 \\
\hline 11 & 8 & 77 & 22 & 33 & 0 & 0 \\
\hline$" 1$ & 9 & 94 & 28 & 34 & 0 & 0 \\
\hline 11 & 10 & 56 & 36 & 32 & 0 & 0 \\
\hline$" 1$ & 11 & 79 & 18 & 29 & 0 & 0 \\
\hline$" 1$ & 12 & 77 & 17 & 30 & 0 & 0 \\
\hline 11 & 13 & 72 & 7 & 3 & 28 & 0 \\
\hline$"$ & 14 & 1 & 81 & 28 & 3 & 0 \\
\hline$" 1$ & 15 & 2 & 59 & 22 & 2 & 0 \\
\hline " & 16 & 40 & 25 & 24 & 0 & 0 \\
\hline$" 1$ & 17 & 0 & 79 & 33 & 0 & 0 \\
\hline " & 18 & 1 & 87 & 32 & 1 & 0 \\
\hline$"$ & 19 & 76 & 14 & 3 & 29 & 0 \\
\hline 11 & 20 & 78 & 10 & 0 & 28 & 1 \\
\hline$" 1$ & 21 & 73 & 6 & 3 & 27 & 1 \\
\hline "1 & 22 & 0 & 27 & 8 & 0 & 0 \\
\hline 11 & 23 & 70 & 15 & 23 & 1 & 0 \\
\hline$" 1$ & 24 & 1 & 52 & 20 & 0 & 0 \\
\hline 11 & 25 & 76 & 11 & 27 & 0 & 0 \\
\hline
\end{tabular}


Table 2. Character of sporophore-branching according to data of various laboratories resulting from anal$y$ sis of international collection of actinomycetes.

\begin{tabular}{|c|c|c|c|c|c|}
\hline \multirow{3}{*}{$\begin{array}{l}\text { Strain } \\
\text { Nos. }\end{array}$} & \multirow{3}{*}{$\begin{array}{l}\text { Total } \\
\text { number of } \\
\text { analyses }\end{array}$} & \multicolumn{4}{|c|}{ Established as verticillate } \\
\hline & & \multicolumn{2}{|c|}{$\begin{array}{c}\text { With primary } \\
\text { verticils } \\
\end{array}$} & \multicolumn{2}{|c|}{$\begin{array}{c}\text { With secondary } \\
\text { verticils }\end{array}$} \\
\hline & & Spiral & Straight & Spiral & Straight \\
\hline 1 & 87 & 9 & 0 & 0 & 1 \\
\hline 2 & 88 & 0 & 2 & 0 & 0 \\
\hline 3 & 87 & 0 & 2 & 0 & 0 \\
\hline 5 & 90 & 0 & 0 & 3 & 0 \\
\hline 6 & 93 & 0 & 1 & 0 & 0 \\
\hline 7 & 113 & 0 & 3 & 0 & 0 \\
\hline 8 & 99 & 7 & 0 & 2 & 0 \\
\hline 10 & 89 & 0 & 3 & 0 & 0 \\
\hline 13 & 90 & 3 & 0 & 0 & 0 \\
\hline 19 & 90 & 2 & 0 & 0 & 0 \\
\hline 20 & 88 & 19 & 0 & 0 & 3 \\
\hline 21 & 79 & 13 & 0 & 5 & 0 \\
\hline
\end{tabular}

Such contradictory data give an impression that the characters given cannot differentiate the species and hence are generally unfit for use in taxonomy.

No less inconsistent are the data on the suitability of the external features for the determination of actinomycetes species, found in the works of various authors. This is also evident in papers published in the journal "Microbiologia" as part of discussion on this subject (Waksman, 1959; Tesic, 1960; Baldacci, 1959; Kosmachev, 1959; and others).

We have made a thorough study of the collection of strains circulated by the Subcommittee. Each strain was studied comprehensively: morphologically, culturally, physiologically and biochemically. Each culture was investigated from the standpoint of its antimicrobial and antagonistic features, etc.

Based on our own experience ingeneral and on the results obtained in processing the above-mentioned collection, we shall attempt to systematize the material collected by the 
Subcommittee and to present our views and explanations concerning the puzzling contradictions and inconsistencies.

In the first place, it is useful to touch upon the question of understanding and representing the characters. What is the taxonomic significance of individual characters?

The scheme suggested by the Subcommittee is based on the subdivisions of the shape of sporophores after Pridham et al., (1958). According to this scheme, the nonspiral sporophores are divided into two types: straight and flexuous, single and fascicled. The spiral sporophores are subdivided into, a) typical spirals with many coils which are stretched or tightly compressed; b) primitive spirals in which the sporophores terminate in an incomplete spiral coil of 0.5-l turn; c) spirals formed by the vegetative filaments of a mycelium, etc.

Actinomycetes having the above-mentioned sporophore structure are divided into sections (Spira, Reticulumapertum).

According to our data such a subdivision has no sufficient experimental basis. One and the same species with nonspiral sporophores may have straight and flexuous, short and long sporogenous branches. Our collection includes more than 350 strains of the same species (Actinomyces streptomycini), a streptomycine producer, collected by us from various soils of the USSR. They are all similar in their physiological, biochemical and antimicrobial properties. Their sporophores are nonspiral, but with some strains they are short and straight, with others long and flexuous; occasional sporophores are curved (but not spiral), they bear a slight resemblance to irregular spirals.

The same strain may have long flexuous or short straight sporophores depending on the conditions of growth and the composition of the nutrient medium.

Similar features are exhibited by other species: $\underline{A}$. oliv-

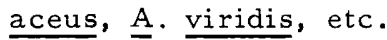

We believe that nonspiral sporophores are all of the "nonspiral" type, no matter whether they are short and straight, long and flexuous, or curved.

There may occur individual species with short, bristly, straight sporophores, sufficiently characteristic and stable, hereditarily fixed. In such cases the given character is correlated with other characters: physiological and biochemical. 
The concept of spiral sporophores is often no longer justified and quite arbitrary. They are either excessively differentiated or insufficiently analyzed, which results in erroneous determination.

Let us consider the so-called "primitive sporophore," i.e. a sporophore with an incomplete terminal coil (0.5-1 turn). According to our observations such sporophores are found consistently only in individual actinomycetes. In such cases this character is always correlated by other characters, and we are inclined to regard it as specific, inherent in definite organisms. As a rule, they ha e no real spiral sporophores with many coils. However, we class such sporophores as spiral.

In the majority of actinomycetes such incomplete coils of spiral sporophores are unstable and occur only occasionally, depending on the conditions of growth, the composition of the medium and other factors. They are often formed in the same culture along with complete and numerous coils. We consider such cultures as having fully formed spiral sporophores. "Imperfect" spirals are not typical of them, and represent a manifestation of polymorphism, not a specific character.

Some actinomycetes form highly characteristic and tightly compressed spirals appearing as a glomerule or a miniature fist on the end of the sporophore. So far we are unable to say to what extent it is a specific characteristic and what its hereditary stability is, since no special investigations have been carried out. We may note, however, that in many cases cultures with such sporophores possess also a number of other distinguishing features of correlative nature. At the same time many strains of actinomycetes having sporophores tightly compressed into fists, do not differ in any respect from strains with conventional spiral spore chains.

In our classification of actinomycetes we regard the above-mentioned curls in the glomerules as spiral formation of sporophores.

Of very vague nature is the spiral coiling of filaments of the vegetative mycelium. It is common knowledge that in cultures of actinomycetes one may observe occasional formation of long "corkscrew"-curling of mycelial hyphae at various sites along its length. Such filaments are not associated either with sporogeny or with any other phase of the culture development. No spores or any other reproducing 


\section{AND TAXONOMY}

elements are found in spirally coiled filaments.

It should be noted that such mycelium curls are observed in mycelial fungi, and sometimes in filiform bacteria. The nature and cause of their appearance are yet to be leamed. Our observations show that this character in actinomycetes is not stable, it far from consistently manifests itself in the same strain, and no relation between it and the conditions of the culture growth can be detected. There are nogrounds as yet to accept it as a taxonomic character in determining and differentiating species.

It is noteworthy that actinomycetes having spiral hyphae in the vegetative mycelium, generally form spiral sporophores. The latter may be rare and may remain unnoticed in a cursory examination of the culture.

In actinomycetes with typical spiral sporophores spirals are not always (and not by everyone) detected in the same culture. For instance, 92 analyses of strain No.10 showed 56 spiral and 36 nonspiral sporophores. Eighty-eight analyses of strain No. 20 are said to have yielded 78 spiral and 10 nonspiral sporophores. Similar contradictory data were obtained with other strains investigated (Table l).

What is the cause of such a contradiction? How shall we regard actinomycetes with such nonuniform characters: shall we class them as spiral or nonspiral cultures?

We believe that if any strain has even a single spiral sporophore on any medium, it should be classed as a spiral culture. It is well known in mycology that very many species form ascospores quite rarely, they usually multiply by means of a vegetative reproduction; many investigators are unable to detect asci in them; nevertheless such species are classed as ascomycetes. As an illustration, we can quote representatives of the species Aspergillus, Penicillium, etc. In brewer's yeast, spores are detected very rarely, the cells multiplyby budding as is usual in asporogenous yeast, and still this yeast is classed as sporogenous and included in the genus Saccharomyces.

Many similar examples could be cited in mycology where the true place of the culture in the system of organisms is established by a single detected sporophore. Similar cases are found in bacteriology. Certain strains of sporogenous bacteria form single spores, and only in strictly definite media and under definite conditions of growth. For instance, some cultures of sporogenous oligonitrophils form spores 
very rarely; to find cells with spores requires careful microscopic study of the preparations, while in many preparations no spores whatever can be detected. Nevertheless, we class such cultures among definite species of sporogenous bacteria, and not among the group of nonsporogenous forms.

The actinomycetes should be no exception.

Based on our long experience we can state that if this or that strain shows even individual spiral sporophores only in certain media or in a single medium, it is possible to achieve a more abundant spiral formation in the future.

We have noted repeatedly that the detection of spirals, like that of any other character, requires definite conditions of nutrition and growth.

Routine laboratory practice frequently reveals, in the same strain, the presence of spirals in some cases and their absence in others; the spirals are more abundant in some media, while others do not exhibit them at all or show them in rare, isolated cases. Some strains form spiral sporophores only in definite media. Sometimes we have to test many nutrient media, change individual medium components, try separate sources of carbon and nitrogen, very the $\mathrm{pH}$ of the medium or the intensity of aeration, the temperature of growth, etc.

There is no single medium for spore formation which would be optimal for all species of actinomycetes. Although most cultures give the highest yield of spiral sporophores on deficient or semideficient synthetic media, on the Czapek medium, SM-l etc., one should not restrict himself to the se in spiral detection. Different media should be tried to make sure that the culture under study can be classed among actinomycetes with nonspiral sporophores.

Some strains form spiral sporophores on a synthetic medium with glucose and maltose; others, with sucrose or mannitol, still others, with glycerol or starch or some other carbon nutrient. Different behavior with respect to nitrogen nutrition is observed. Some strains form spirals preferably on synthetic media with nitrate nitrogen, others, with ammonia nitrogen.

Many or most actinomycetes have abundant spiral sporophores on deficient (water-agar) or semideficient (SM-l or Czapek, Ashby-agar with $0.2 \%$ carbohydrate) media. Cultures grow very slowly on these media. Often their 
growth cannot be discerned by the unaided eye. There is growth, however; using a microscope, one can see the development of mycelium filaments creeping over the surface of the agar, and numerous sporophores branching off from the mycelium.

The process of sporogeny and the sporophore formation is greatly affected by the composition of the mineral salts used for the media. Even tap water may influence this process. It is well known that the composition of tap water varies markedly depending on the season. In spring it is not the same as in summer or in winter. It is also different from reservoir to reservoir. The water composition of mountain rivers differs from that of rivers flowing through lowlands and particularly through swampy areas.

Very substantial influence is exerted on sporogeny by trace elements contained in nutrients.

The optimal temperature for sporophore and spore formation in actinomycetes lies between 20 and $30^{\circ} \mathrm{C}$ (it is considerably higher for thermophils). At $37^{\circ} \mathrm{C}$ sporogeny is slow, while many species do not exhibit it at all, the colonies remain bare or reveal sterile aerial mycelium. Abundant formation of spores and spiral sporophores is observed at $15-20^{\circ} \mathrm{C}$, but it is slower than at $25-30^{\circ} \mathrm{C}$ (Krasil'nikov, 1938).

Some actinomycetes react to the composition of the glass vessel in which they are growing. New or poorly washed (unleached) test tubes or retorts may produce marked changes in the character of sporogeny. It is not a rare occasion that two closely spaced test tubes containing identical cultures planted at the same time show an abundance of sporophores in one test tube and little or none in the other.

In our earlier investigations we showed (1938-59) that cultures for which spiral sporophores are not typical, never form them, and vice versa. This may be illustrated by strains of the globisporine group which have been thoroughly studied by us.

Our laboratory surveyed more than a thousand cultures of this group of actinomycetes. They have been classed into 8 species, all having nonspiral sporophores. Six hundred fifty strains belonging to these 8 species have been subjected to special investigation. In these we have never observed spiral sporophores or sporophores with even $0.5-1$ 
coil. We grew them in various media: in deficient or semideficient synthetic media or in rich complex, albuminous media, in natural vegetative substrates (carrots, potatoes, etc.); in all cases the cultures had typical nonspiral sporophores.

We have reported earlier (1958) that these cultures were subjected to experimental mutability. Under the effect of various mutagenic influences and spontaneously, many variants were obtained which differed from the original and among themselves in physiological and cultural characters; there were variants with a different nature of colony growth, with another shade in the colouring of the aerial mycelium; we had strains which were entirely devoid of aerial mycelium, and also variants differing in the assimilation of carbohydrates, in enzymatic and other properties. However, we have never noted cultures which changed the shape of the sporophores, i.e. turned from nonspiral to spiral cultures.

We carried out similar investigations of experimental mutability with certain cultures forming typical spiral sporophores-A. violaceus, A. coelicolor. These organisms form numerous and well-pronounced spiral sporophores with 3-6 coils. We tried to make them produce mutants with straight sporophores. In the process of experimental variability from violet actinomycetes we have obtained more than 50 mutants: some of them are colourless, others are coloured definitely red (not violet), forming a single red or pink pigment; there were mutants without aerial mycelium with typical black-violet bare colonies, also mutants which did not colour the medium, i.e. those forming chromophorous pigments (which are not excreted into the medium). Many mutants featured varying intensity of proteolytic, amylolytic and other fermentation activity. We had strains with rare spiral sporophores which, in addition to that, formed them by no means in all media. However, we did not obtain any variant entirely lacking in ability to form spiral sporophores.

A blue actinomycete, A. coelicolor, yielded about 250 variants: pigmented and colourless, with and without aerial mycelium, variants with different colour shades, chromoparous and chromophorous; many variants had biochemical and enzymatic distinctions. But there was not a single strain which would change the type of sporogeny and acquire typical nonspiral sporophores. Similarly to the preceding 
cases, there were strains with rare spiral sporophores formed only in definite media. However, they possessed typical spirals.

The foregoing shows that the shape of sporophores in actinomycetes is a. sufficiently stable, hereditarily fixed and always clearly pronounced character under proper conditions. Consequently, this character is also most suitable in taxonomy when establishing species, groups or subgroups.

In our opinion, the data presented here explain the contradictory results obtained by various investigators in the study of the international actinomycete collection. In all cases where obviously spiral actinomycetes did not exhibit spiral sporophores, the strains were not studied thoroughly enough. In some cases the variability and polymorphism of cultures were not taken into account. This is confirmed by our investigations.

Considering such a great divergency of results of the above-mentioned investigators, we placed particular emphasis on studying the same actinomycete collection. At first we strictly adhered to all the methods of culture studies recommended by the Subcommittee. We used the media and the conditions of growth recommended in the instructions.

We were not satisfied with results obtained and worked out an extended program of investigations, particularly with regard to those strains that yielded dubious results. For spore formation and culture growth we used synthetic media: Czapek, SM-1, SM-III starch-ammonium agar, also potato agar, wort agar, etc. Of the deficient media we used tap water $+1.5 \%$ agar-agar, semideficient media of A shby, SM-1 and others.

We studied 24 strains. One strain, No. 24 , ceased to grow in subculture and perished. Strain No.22 represents a proactinomycete, and was not correlated with the other strains in the general analysis.

Each strain was subjected to $2-3$ parallel tests, while doubtful strains underwent 4-5 parallel tests. All cultures were analyzed three times according to their growth stages, i.e. on the 10 th, 15 th, and 20 th days. Hence, each strain was examined microscopically 10-15 times, some even more. The results are given in Table 3 .

It can be seen that 12 strains, Nos. 4, 5, 8, 9, 11, 12, 13, 19, $20,21,23$ and 25 have spiral sporophores in almost all the media tested, the spirals being well pronounced, numerous 
in each culture, with 2 to 5 or more coils. They may be extended or tightly compressed. These data agree with tho se of most other foreign laboratories. All these strains clearly belong to spiral forms.

Strains No.14,17 and 18 cause no difficulties. They do not form spiral sporophores in any of the test media. Their sporogenous branches arestraight or flexuous, short or long, without any spiral coils. Many foreign investigators also have noted the absence of spirals.

The remaining 8 strains are less well defined, and the data on them most contradictory. Therefore we centered our attention on these strains and studied them most thoroughly. Each strain was subjected to special analysis and detailed microscopic examination. Finally we established that all these cultures belonged to the class of actinomycetes with scant fructification. They very rarely form spiral sporophores, and only in individual cultures single spirals can be detected after a long examination of the Petri-dish with the culture.

Strain No.l showed individual spiral with $1-3$ coils only on two media: in the Czapek medium with maltose and in a starch-ammonium agar. In strain No. 7 such a spiral formation was observed only in one medium, starch-ammonium agar. In strain No.10 spirals with complete coils of $1-3$ turns were noted only on SM-1 medium with sucrose. The spirals were very rare and could not always be detected. Most frequently the sporophores terminated in incomplete spiral coils of 0.5-1 turn. Such sporophores often form in other tested media.

In strain No.15 individual spiral sporophores are found on a CPI medium with sucrose. On the other media the sporophores are nonspiral, long curved or flexuous, but not coiled into spirals.

In strains No.2, 6 and 16 the sporophores terminate in an incomplete spiral or a coil of 0.5-1 turn. Such formation of sporophores is revealed in many media and is rather abundant. Our attempts to obtain real spiral sporophores in these strains failed.

Strain No. 3 does not form spiral sporophores on any of the media tested. It has long and drastically curved sporogenous branches which often resemble spiral coiling; we did not, however, observe real spirals. It is probable that some investigators took such curved branches for spirals. 
Page 145

BACTERIOLOGICAL NOMENCLATURE AND TAXONOMY

Table 3. Formation of spiral sporophores in actinomycetes on various media (International Collection). Author's observations.

\begin{tabular}{|c|c|c|c|c|c|c|c|c|}
\hline $\begin{array}{c}\text { Strain } \\
\text { No. }\end{array}$ & $\begin{array}{l}4 \\
0 \\
0 \\
0 \\
1 \\
4 \\
4 \\
0 \\
4 \\
3 \\
3 \\
3\end{array}$ & 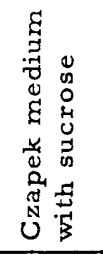 & 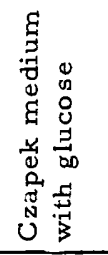 & 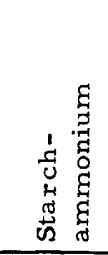 & 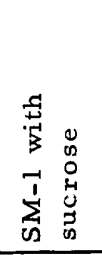 & $\begin{array}{l}4 \\
0 \\
00 \\
0 \\
0 \\
+0 \\
+0 \\
0 \\
0 \\
0\end{array}$ & 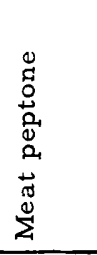 & 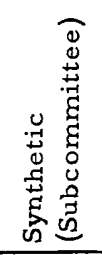 \\
\hline 1 & - & - & + & + & - & - & - & - \\
\hline 2 & - & - & - & + & - & - & - & - \\
\hline 3 & - & - & - & - & - & - & - & - \\
\hline 4 & +++++ & ++++ & +++++ & +++++ & +++++ & ++++ & + & +++ \\
\hline 5 & +++++ & +++++ & +++++ & +++++ & +++++ & +++ & ++ & +++++ \\
\hline 6 & + & - & + & + & - & - & - & - \\
\hline 7 & - & + & - & ++ & + & - & - & - \\
\hline 8 & +++++ & +++++ & +++++ & ++++ & +++++ & ++ & + & ++ \\
\hline 9 & +++++ & +++++ & ++++ & +++++ & ++++ & ++++ & ++++ & +++++ \\
\hline 10 & - & tt & + & + & ++ & + & - & + \\
\hline 11 & +++++ & +++++ & ++++ & +++++ & ++++ & $+t+++$ & - & +++ \\
\hline 12 & ++++ & +++++ & +++++ & ++++ & +++++ & ++++ & + & ++ \\
\hline 13 & ++++ & ++++ & $+++t$ & +++++ & - & $++t$ & - & ++++ \\
\hline 14 & - & - & - & - & - & - & - & - \\
\hline 15 & - & - & - & - & + & - & - & - \\
\hline 16 & + & + & + & - & + & - & - & - \\
\hline 17 & - & - & - & - & - & - & - & - \\
\hline 18 & - & - & - & - & - & - & - & - \\
\hline 19 & ++++ & ++++ & ++++ & +++++ & ++++ & ++++ & ++ & ++++ \\
\hline 20 & +++++ & +++++ & +++++ & +++++ & ++++ & +++++ & ++ & ++++ \\
\hline 21 & ++++ & $++++t$ & ++++ & +++++ & + & ++++ & + & ++ \\
\hline 22 & - & - & - & - & - & - & - & - \\
\hline 23 & ++++ & +++++ & ++++ & ++++ & ++++ & +++++ & +++ & +++++ \\
\hline 25 & ++++ & ++++ & +++++ & +++++ & ++++ & ++++ & + & +++ \\
\hline
\end{tabular}


Thus, the study of the international collection of actinomycetes can be summed up as follows: of the 24 strains investigated by us 19 represent organisms with spiral sporophores, 4 ,constitute the group with nonspiral sporophores and $l$ is a proactinomycete.

Among the first 19 strains 12 have abundant complete spiral sporophores produced in all or most media, 4 strains $(1,7,10$ and 15$)$ very rarely form spiral sporophores with $1-3$ coils and even then not on all media; 3 strains 2, 4, 16) have incomplete spirals with $0.5-1$ coil on the sporophore end.

Among the 4 strains of the second group (with straight sporophores), strain No. 3 has curved but nonspiral sporophores. In cursory examination they may be taken for spiral forms.

\section{Spore Structure in Actinomycetes}

The structure and form of spores are an essential character in actinomycetes. We have reported earlier that spores in actinomycetes may be of dual structure. In some cases they are spherical and oval in shape; in others rodlike and cylindrical.

Further investigations have shown that actinomycetes with spherical and oval spores are found more often. Not infrequently strains with spherical spores simultaneously have elongated or even rod-like spores. At times the same sporophore branch bears both spherical and elongated spores. Such spores are observed in many representatives of the globisporine group and even in one and the same species. For instance, A. streptomycini may exhibit spherical, oval and elongated spores at the same time. There are cultures, however, whose spores have only a rodlike or cylindrical shape. In such cultures this character is rather constant and may serve in the differentiation and identification of the species.

The mode of spore formation is an essential character. Previously we showed that cultures of some actinomycetes form spores by fragmentation, in others by segmentation. In the first case the protoplast of the sporogenous branch breaks up into separate sections, exhibiting a chromatinous substance and protoplasm strongly stained by basic dyes. These sections become compacted, rounded up and develop 
into spores. The hyphal wall does not disappear. The spores are inside the hypha. Later on the hyphal wall dissolves or bursts and the spores are released (Krasil'nikov, 1938).

In segmentation, spores are formed by simple division of the sporogenous branch with the aid of cross partitions. The latter are formed in a usual way simultaneously along the whole sporophore. Then such a hypha breaks up into separate cell-spores. In such cases the newly formed spores have a cylindrical shape with sharply cut ends. Later they may round off. If the spores are short, they become oval or spherical; if the spores are long, they become elongated with rounded ends. Consequently, when determining the shape of the spore, one should know their original shape immediately after the breakup of the sporophore.

Cultures forming spores by segmentation are found rarely and it is only natural that they are not so well investigated as cultures with fragmentation spores. Our data indicate that such organisms should represent special species or groups of actinomycetes. It is worth mentioning that segmentation spores are typical of many proactinomycetes. In this respect, the actinomycetes show great similarity to proactinomycetes. As shown by our investigations, this similarity is not only external, but also phologenetic.

The relationship between the actinomycetes and the proactinomycetes was established by experimental variability (Krasil'nikov, 1939) as well as by finding intermediate forms in nature. We described strains which could be equally classed as actinomycetes or proactinomycetes on the basis of their structure and development.

In recent years much attention has been given to the structure of the spore wall. In electron microscope investigations, Kriss et al. (1945), Carvajal (1946), and Bringman (1951) found the surface of actinomycete's spores to be smooth and not exhibiting any outgrowths.

More recently Flaig (1952), then Kuster (1953), Nikitina (1957), Ettlinger et al. (1958), Baldacci (1953-60), and others established that actinomycetes may have spores with a smooth membrane and spores with outgrowths. These outgrowths may be of different types: warty, prickly, needlelike, filamentous; they may also be long or short.

Some investigators are convinced that these outgrowths are sufficiently characteristic to be used for classifying actinomycetes. They established special sections on the 
basis of the shapes of these outgrowths (Ettlinger et al., 1958; Flaig and Kutzner, 1958; Preobrazhenskaya et $\bar{a}$.., 1959). The sections established by them included variegated species, often differing greatly. For instance, in the same section Ettlinger includes the producers of streptomycin, A. streptomycini, A. aureus, A. candidus, A. coelicolor, $\bar{A}$. ruber, A. olivaceus. Accor ding to Preobrazhenskaya (1959) this procedure is unsuitable for grouping actinomycetes. The material presented by them shows that the same series includes cultures with smooth, prickly, hairlike and needlelike outgrowths. The resumé, however, presents an opposite conclusion.

Analyzing the published data and our own observations, we may consider two types of spores: smooth and prickly, the same strain may have different outgrowths: prickly, needlelike, hairlike and tuberclelike, depending on the conditions of culture growth.

In studying our collection of actinomycetes Leshevalier and Tihonienko (1960) showed that in some cases individual smooth spores are found along with prickly spores in the same preparation and on the same sporophore. However, the se individual spores are an exception.

The cultures studied show a definite relationship between the shape of the spores and their species group. Nikitina (1957) established that actinomycetes of the globisporus group have smooth spores. In the violet group we have studied 45 strains belonging to several different species. In one species ( 3 strains) the spores had a smooth surface, in all others ( 39 strains) they proved to have outgrowths.

We noted prickly spores in some species of blue actinomycetes, one species of the green group. A. viridis, also in $A$. viridichromogenus; in actinomycetes of the orange group (35 strains, 5 species) we observed spores with a smooth membrane.

At this juncture we cannot tell whether such a relationship between the spore shape and the species group is inherent in all actinomycetes. The data available, however, point to the fact that this character may serve the purposes of differentiating the organisms under study. Yet, it is suitable only for subdividing the species within definite, outwardly uniform groups, rather than accidentally selected sections or series.

Leshevalier and Tihonienko have shown that this character 
is stable, it is manifested under various growth conditions, in various media. They tested 29 media in which they cultivated several strains of various actinomycete species. The spores were investigated at different stages of growth and development of the cultures after 3,10,16 and 20 days. Prickly spores were found in A. coelicolor (strains 1047, 2078), A. violaceus (strains 829, $1144,2525,2522,2069,2587$, $117,7 \overline{9}$ ) and A. viridichromogenus (strains 93 and 94).

Their data show that the spore surface does not change with the composition of the medium: in strains with smooth spores the latter remain unchanged, and prickly spores retain the prickles under different conditions of nitrogen and carbon nutrition. Only in individual cases have they observed spores with a smooth membrane along with prickly spores.

The authors managed to trace the sequence of the formation of prickles on the membranes. According to their data, the prickles form after the spores have acquired a sufficiently definite shape and have begun to ripen. Our observations show that they form at the expense of the external layer of the wall.

The material presented here gives us grounds to believe that the presence or absence of outgrowth on the spore surface may be used as a differentiating character when grouping actinomycetes. Two types of spores may be regarded as established: smooth spores and spores with prickly and other outgrowth.

Assuming the external structure of the spores to be a taxonomic character, we can use it only in combination with other characters when identifying and establishing actinomycete species. This character can be used successfully when distinguishing species within outwardly uniform groups.

\section{On Verticils in Actinomycetes}

The tables recommended by the Subcommittee include characters relating to the types of arrangement of sporogenous branches. They single out monopodial sporophores, i.e. those alternating on the mycelium filaments; followed by the verticillate arrangement, the verticils being subdivided into primary and secondary. The sporophores in these verticils may be straight or spiral. Thus, 5 types of formation arrangements of sporophores are differentiated. Data on the arrangement of the sporophores are presented 
in Table 2. They are less contradictory than the data on the structure of sporophores and spores.

It should be noted that this character cannot as yet be used to differentiate species or groups of actinomycetes. It is still far from clearly understood and we cannot state the degree to which it is stable and hereditarily fixed. Our observations of many years standing show that almost all (or even all) cultures can form verticils under certain conditions of growth and in certain nutrient media. In some groups, however, verticils are found more often and more consistently than in other representatives of the actinocetes. For instance, verticils are usual in orange actinomycetes, they are formed on many or all media where air mycelium is present. Less frequently, they are observed in actinomycetes of the red and yellow-brown groups. They are rare in the cultures of the grey, white, blue and other groups.

The nature of the branching and arrangement of the sporophores may be used only for additional characterization of the strains, rather than for the determination and identification of the species.

All these data are of a very relative nature. Orange actinomycetes can develop in media without the formation of verticils, or the latter may be detected rarely, as in many grey or white actinomycetes.

\section{Culture Colouration}

As is well known, cultures of many actinomycetes display different colours. Colouration is characteristic of aerial mycelium, colonies and nutrient media. Not infrequently colouration affects only aerial mycelium, or, more precisely, spores, whereas colonies and media remain colourless. Aerial mycelium in pigmented cultures often differs in colour from those of colonies or of media. For example, in blue actinomycetes aerial mycelium may have whitish-grey, light grey, bluish, greenish-blue, bluish-grey, bluish-brown or some other colour. The colonies and the medium are usually blue or dark blue in colour. An acidified medium turns red. Pigments in this actinomycetes group belong to the anthocyanin type; as litmus, they change their colour with change in the $\mathrm{pH}$ of the medium.

Culture pigmentation is an essential and highly expressive 


\section{AND TAXONOMY}

taxonomic character, and all investigators attach much importance to it. Some authors, however, attach more importance to the colouration of aerial mycelium and often class actinomycetes into sections and series according to this characteristic (Baldacci et al., 1953; Hesseltine et al., 1954; Flaig and Kutzner, 1958; Yamaguchi and Saburi, 1955 ; Ettlinger et al. 1958; and others). In the USSR a group of workers of the Prof. Gauze Laboratory differentiates actinomycetes by the colouration of air spores (see Collection of articles on classification of actinomycetes, 1957).

The Subcommittee recommendations quote the definite shades of the colouration of aerial mycelium and the colony (substrate mycelium). There is a great divergency in the data on the se characters presented by different laboratories; the discrepancies are particularly pronounced in the data on the colouration of aerial mycelium. There is not a single strain with identical colour characters; 5 or 6 colours are given for each strain, certain strains showing 8-9 colours of the aerial mycelium. For instance, data of 118 analyses performed by different laboratories are presented with respect to strain No.12. Of these, 27 characterize aerial mycelium as white, 1 as yellow, 1 as orange, 9 as pink, 3 as red, 3 as green, 60 as grey, and 11 as brown; i.e. here we have almost all possible colours. Similar diversity is observed in the remaining strains. Table 4 indicates aerial mycelium colouration for 17 strains; the colourations of the other strains coincide with those of the neighboring cultures.

The Subcommittee gives quite vague indications regarding the colouration of substrate mycelium (colonies). The Subcommittee table (Table 5) quotes colours; white, grey, colourless (colonies), then yellow, orange, red, violet, blue, green, brown and black. Nearly all strains were assigned $5-6$ colours of the colonies. For instance; 121 analyses of strain No. 1 yielded in 18 cases colourless colonies, in 30 white, in 33 yellow, in 4 orange, in 7 grey, in 29 brown. For strain No. 11 the colour of the colonies is given: as colourless in 13 cases, as white in 18 , as yellow in 24 , as otange in 5, as red in 1 , as violet in 4 , as green in 1 , as grey in 17, as brown in 34, and as black in 1 . Similar diversity is observed with the other strains.

Having analyzed the tables presented by the Subcommittee and the results of our own investigations, we come to the conclusion that the diverging indications noted by different 
Table 4. Colouration of aerial mycelium of cultures from the international collection based on data from various laboratories.

\begin{tabular}{|c|c|c|c|c|c|c|c|c|c|}
\hline 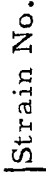 & $\begin{array}{l} \pm \\
. \\
3 \\
3 \\
\end{array}$ & $\underset{0}{3}$ & $\begin{array}{l}0 \\
0 \\
0 \\
E \\
0 \\
0 \\
0 \\
\end{array}$ & 艺 & $\begin{array}{l}00 \\
0 \\
\underline{0}\end{array}$ & $\stackrel{0}{\stackrel{g}{g}}$ & $\begin{array}{l}\tilde{D} \\
0 \\
0 \\
0 \\
0\end{array}$ & 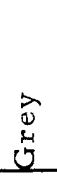 & $\begin{array}{l}z \\
3 \\
0\end{array}$ \\
\hline 1 & 78 & 4 & - & 3 & - & - & - & - & 3 \\
\hline 2 & 64 & 22 & - & - & - & - & 3 & 24 & 6 \\
\hline 4 & 32 & 4 & - & 12 & 6 & - & - & 54 & 11 \\
\hline 6 & 17 & 19 & - & 2 & - & - & 2 & 66 & 11 \\
\hline 7 & 31 & 9 & 1 & 2 & - & - & - & 54 & 5 \\
\hline 10 & 35 & 2 & 6 & 36 & 7 & - & - & 4 & 31 \\
\hline 12 & 27 & 1 & 1 & 9 & 3 & 3 & 3 & 60 & 11 \\
\hline 13 & 10 & - & - & - & - & 27 & 37 & 51 & 2 \\
\hline 14 & 51 & 30 & - & 25 & 4 & - & 4 & 1 & - \\
\hline 15 & 36 & 7 & 1 & - & 1 & 6 & 1 & 40 & 2 \\
\hline 16 & 26 & 45 & - & - & - & - & 4 & 15 & 1 \\
\hline 17 & 62 & 21 & 1 & - & - & - & 8 & 10 & 11 \\
\hline 19 & 40 & - & - & 48 & 17 & 5 & - & 3 & - \\
\hline 20 & 15 & 2 & - & - & - & 35 & 37 & 42 & - \\
\hline 21 & 13 & 1 & - & - & - & 27 & 32 & 54 & 2 \\
\hline 23 & 34 & 3 & - & 6 & - & 1 & 1 & 45 & 13 \\
\hline 25 & 40 & 1 & 2 & 24 & 4 & - & - & 32 & 8 \\
\hline
\end{tabular}

authors with regard to the pigmentation of the colonies are the result of a subjective evaluation and often give an erroneous characterization of the colour.

It serves no purpose to subdivide the actinomycete colonies into colourless, white and grey. There can be no such differentiation. A colony of an actinomycete (substrate mycelium) can never be white or grey. It can be colourless, i.e. having no special colour. Greyish, or more precisely, dingy colonies are typical of all colourless actinomycetes. they may be more or less bright, depending on the composition of the medium. Our observations show that colourless, white and grey colonies are of the same order of colouring. 
Page 153

BACTERIOLOGICAL NOMENCLATURE

AND TAXONOMY

Table 5. Colour of actinomycete colonies from international collection on the basis of data from various laboratories.

\begin{tabular}{|c|c|c|c|c|c|c|c|c|c|c|c|c|}
\hline 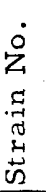 & 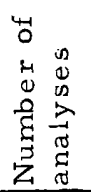 & $\begin{array}{l}\text { un } \\
0 \\
0 \\
-1 \\
3 \\
3 \\
0 \\
0 \\
0 \\
0\end{array}$ & $\begin{array}{l}\stackrel{0}{ \pm} \\
\stackrel{2}{3} \\
3\end{array}$ & $\begin{array}{l}3 \\
\underset{0}{0} \\
0 \\
\end{array}$ & 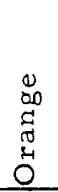 & $\begin{array}{l}00 \\
0 \\
\text { د }\end{array}$ & $\begin{array}{l}\stackrel{\vec{\nu}}{\overrightarrow{0}} \\
\stackrel{0}{\circ} \\
\stackrel{-1}{>}\end{array}$ & $\frac{0}{\Xi}$ & 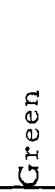 & $\begin{array}{l}\lambda \\
0 \\
0 \\
0 \\
0\end{array}$ & 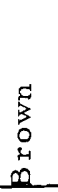 & $\begin{array}{l}\frac{x}{0} \\
\frac{\pi}{\vec{m}} \\
0\end{array}$ \\
\hline 1 & 112 & 18 & 30 & 33 & 4 & - & - & - & - & 7 & 29 & - \\
\hline 2 & 103 & 3 & 3 & 43 & 2 & - & - & - & 1 & - & 47 & 4 \\
\hline 3 & 156 & - & 7 & 44 & - & 1 & - & - & 31 & 28 & 21 & 21 \\
\hline 4 & 132 & 21 & 32 & 10 & 6 & 19 & - & - & - & 25 & 19 & - \\
\hline 5 & 131 & - & 1 & 24 & 8 & 10 & - & - & 6 & 12 & 69 & - \\
\hline 6 & 93 & - & - & 67 & 8 & 1 & - & - & 3 & 2 & 12 & - \\
\hline 7 & 114 & 14 & 20 & 38 & 6 & 3 & - & - & 2 & 16 & 13 & - \\
\hline 8 & 123 & 20 & 34 & 42 & 2 & - & - & - & - & 19 & 6 & \\
\hline 9 & 132 & 11 & 25 & 52 & 14 & - & - & - & - & 3 & 27 & - \\
\hline 10 & 117 & 28 & 21 & 14 & 9 & 8 & 1 & - & - & 8 & 28 & - \\
\hline 11 & 123 & 23 & 18 & 24 & 5 & 1 & 4 & - & 1 & 17 & 34 & 1 \\
\hline 12 & 119 & 8 & 15 & 8 & 4 & 5 & 4 & - & 1 & 19 & 55 & - \\
\hline 13 & 154 & - & - & - & - & - & - & 3 & 43 & 32 & 31 & 45 \\
\hline 14 & 123 & - & 2 & 42 & 49 & 27 & 3 & - & - & - & - & - \\
\hline 15 & 92 & 29 & 10 & 3 & 1 & - & - & 3 & 2 & 28 & 11 & 5 \\
\hline 16 & 111 & 34 & 5 & 26 & - & - & - & - & 6 & 10 & 14 & 16 \\
\hline 17 & 109 & 13 & 12 & 57 & 1 & - & - & - & 2 & 3 & 21 & - \\
\hline 18 & 116 & 19 & 6 & 10 & - & 3 & 23 & 4 & - & 21 & 30 & - \\
\hline 19 & 97 & - & 2 & 1 & - & 51 & 44 & - & - & 3 & - & - \\
\hline 20 & 112 & 6 & 7 & 56 & 9 & 1 & - & - & 2 & 4 & 27 & - \\
\hline 21 & 127 & 3 & 1 & - & - & - & 2 & 3 & 27 & 29 & 39 & 23 \\
\hline 23 & 91 & - & 1 & 24 & 2 & - & - & - & - & - & 60 & 4 \\
\hline 25 & 103 & 3 & 14 & 3 & 24 & 41 & - & - & - & 4 & 14 & - \\
\hline
\end{tabular}


Quite often colourless colonies have a yellow or brownish appearance on the lower side (yellowish-brownish lining). Such cultures have no real pigmentation, being, in fact, colourless forms.

It is entirely improbable that colourless cultures should have such diverse pigmentation as that shown in the table. When noting some culture colouration or other, investigators do not attempt to establish whether it is due to a real pigment or to secondary substances of nonpigmentary nature. Only such colony colouration is suitable for the purposes of taxonomy and differentiation of species which is due to a real pigment, i.e. a metabolite with definite staining and physico-chemical properties.

The colouring of culture-colonies and aerial mycelium varies markedly depending on the composition of the medium and the conditions of growth (Krasil'nikov, 1938, 1949-60). It is most characteristic and pronounced in synthetic media. In these media pigments have a colouring without impurities (brown, chestnut, yellowish, etc.). In complex and especially in albuminous media pigments are less pronounced; often they do not manifest themselves at all or mix with various supplementary substances which alter the basic colour of the pigment.

In certain species pigmentation is particula rly pronounced when individual elements are added to the medium. For instance, in actinomycetes of the green group the pigment is very prominent in the presence of iron citrate. In these cultures iron strongly stimulates the synthesis of the green pigment.

In blue actinomycetes which have, for one reason or other, lost their ability for synthesizing pigment, biotin, if added to the medium, completely restores it, and in weakened strains pigmentation markedly increases. In violet strains with weakened pigmentation the synthesis of the pigment is enhanced under the influence of vitamin $B_{12}$ (Krasil'nikov and Egorova, 1960):

Rather often actinomycete cultures exhibit pigmentation most vividly in synthetic media with an addition of extracts from yeast or corn, oatmeal and other vegetative residues.

We carried out a series of special experiments with a view to establishing the colouring of the actinomycetes studied. The latter were grown on different nutrient media; simple synthetic and complex organix, albuminous: the 


\section{B ACTERIOLOGICAL NOMENCLATURE \\ AND TAXONOMY}

Czapek medium with glucose and sucrose, starch-ammonium, glycerol-asparagine, etc., then media with an addition of oatmeal and corn flour, a medium with a yeast extract, with meat-infusion broth, etc.

Our investigations have shown that there are 6 colourless strains in this collection (Nos. 1, 8, 9, 10,17,20) and 3 doubtful strains ( 11 and 12 ). They are being subjected to further study. One strain (No.2) belongs to the globisporus group, it is also colourless. Three strains $(6,20,23)$ belong to the group of fluorescent actinomycetes, they form a lemonyellow pigment diffusing into the medium. Two strains (13, 21) have a brown-green pigmentation of the colonies and the medium, and two $(3,5)$ have a doubtful similar colour. They stand closer to the group of brown-green actinomycetes. Two strains $(7,16)$ have a definitely yellow colouring of the colony, not of the medium; two have a red-orange or redpink colour $(4,14)$; two have a blue colour $(15,18)$ and two have a violet pigmentation.

To sum up, we can say that among the external morphological and cultural characters, the former are most significant, namely the structure of sporophores and spores. Only such culture colouring can be used successfully which is due to real pigments not to secondary accidental products of metabolism. The most stable pigmentation is that of substrate mycelium (colony). Of essential significance for the differentiation of actinomycete cultures is the ability of pigments to diffuse into the medium. This gives some idea of certain physico-chemical properties of pigment substances.

Less reliable is the colouring of aerial mycelium. However, this too, may be of some significance in differentiating actinomycetes; in individual cases it is sufficiently characteristic of certain groups. For instance, the green colouring of aerial mycelium is inherent only in definite species.

No matter how essential morphological or cultural indications may be, they can not serve as the sole basis for building up the taxonomy of organisms. As we repeatedly emphasized (1939-60), the classification and taxonomy of actinomycetes should be based on a combination of characters: morphological, cultural and physiologic-biochemical. Of great importance in the differentiation of species and, consequently, in taxonomy, are antimicrobial properties and the specificities of their antagonisms (Krasil'nikov, 1950-60). 
The structure of sporophores and spores, as well as culture pigmentation, each taken separately, cannot characterize species since these indications are too general. One and the same structure type of sporophores or spores is characteristic of many entirely different species.

Spiral sporophores are found in representatives of blue, violet, yellow, colourless and other groups of actinomycetes. They cannot be combined into a single species or into a single group by the type of sporogeny.

The same is true of pigmentation as a character. One and the same type of pigment, one and the same culture colouring may be encountered in different actinomycete species.

We agree with Baldacci that the pigmentation of cultures, notably that of vegetative mycelium (colonies) and the medium may be useful in the preliminary, very tentative grouping of actinomycetes, but not in establishing taxonomic units.

In our first manual of ray fungi (1941) the ar ltures are arranged in a definite order by groups in accordance with their pigmentation: starting with blue actinomycetes followed by violet, red, yellow, green, orange, etc. Having no intention to assign taxonomic significance to this group, we did not dare then to use the term "group" to denote the above-mentioned sections. We did not supply the corresponding headings.

At present considerations of purely practical convenience make us use the terms "group" and "subgroup" for certain cultures having identical pigmentation indices and the structure of sporophores and spores. We do not yet assign these groups the significance of a species, subspecies or superspecies, or larger taxonomic unit. A group of outwardly uniform strains may include a few species differing in biochemical, physiological and antimicrobial properties and the specificity of antagonism.

Groups are built, not according to a single character, but according to their combinations. These characters may be different from case to case. For instance, in colourless actinomycetes having white aerial mycelium we observe a group with straight sporophores and a group with spiral sporophores. Green, violet and blue groups of actinomycetes are formed by the solubility of the pigments and by the nature of fructification. It is quite possible that there 
are also other external characters which might be used for the same purpose. Further investigations should be carried out with the aim of establishing new groups and their species. All strains should be thoroughly studied in each group.

After a careful study we classified the globisporus group into 8 species, in violet actinomycetes we detected 7 species and 2 subspecies, the white-blue group clearly exhibits 2 species, the brown-green group also 2 species, the fluorescent group 3 species, etc. (see collection of papers "Biology of Individual Groups of Antibiotic-Producing Actinomycetes 1960").

The actinomycete group enables one, using the uniformity of external indications as a guiding principal, to make a clearer distinction of the great diversity of cultures isolated from natural substrates.

\section{REFERENCES}

Baldacci, Elio. 1959. Extension of Actinomycete classifition. Mikrobiologia. Vol.28, No.2.

- 1956. Antibiotic activity and othe $\mathrm{r}$ biochemical characteristics in the classification of species in actinomycetes. Giorn. Microbiol. 2:50.

- 1958. Criteria for the improvement of the classification of actinomycetes. Internatl. Congr. Microbiol. Proc. 7th Congr. Stockholm.

, J. Comaschi, et al. 1953. General criteria for the systematics of genera and species of Actinomyces and Micromonospora. Symposium Actinomycetales. Internatl. Congr. Microbiol. 6th Congr. Rome. p. 20.

, E. Gibardi, and A. Amici. 1956. Il Ciclo di vita degli actinomyceti osservato al microscopio electronico. Giorn. Microbiol. lc:512.

-A. Grein, and C. Spalla. 1955. Studio di und "seria" di Specie di actinomiceti $\underline{B}$. diastaticus. Giorn. di Microbiol. I:127.

Bringman, G. 1951. Electronenmikroskopische und lichtmikroskopische Studien zur Morphologie und Entwicklung von Streptomyces griseus Krainsky. Zentralbl. Bakt. Parasitenk. i, Bd. 157. S. 349.

Carvajal, F. 1946. Studies on the structure of Streptomyces griseus. Micologia 38:587. 
Ettlinger, L., R. Corbaz, and R. Hutter. 1958. Zur Systematic der Actinomyceten. Arch. f. Mikrobiol. Bd. 31 , S. 326 .

Flaig, W., H. Bentelspacher, E. Küster, and G. Segler Holzweissig. 1952. Beitrage zur Physiologie und Morphologie der Streptomyceten. Plant and Soil. Bd.4, S. 118 .

, E. Küster, and H. Bentelspacher. 1955. Elektronenmikroskopische Untersuchungen an Sporen verschiedener Streptomyceten. Zentralbl. f. Bacter. Parasitenk. II. Bd. 108 . S. 376 .

and H. Kützner. 1958. Beitrag zur Systematic und

Okologie der Gattung Streptomyces Waksman et Henrici Dissertation.

Gauze, G.E., Preobrazhenskaya et al. 1957. Problems of Classification of Actinomycete-Antagonists, Moscow.

Hesseltine, C., R. Benedict, and T. Pridham. 1954. Useful criteria for species differentiation in the genus Streptomyces. Ann. New York Acad. Sci. 60:136.

Kosmachev, A.E. 1959. Role of thermophily in actinomycete classification. Mikrobiologia. 29(6).

Krasil'nikov, N.A. 1938. Ray Fungi and Related Organisms. Moscow.

- 1941. Manual of Ray Fungi. Moscow.

- 1949. Manual of Bacteria and Actinomycetes. Moscow.

. 1951. On Intra- and interspecies antagonism in actinomycetes. DAN SSSR, 77(1).

. 1956. On classification of antibiotic-producing actinomycetes. Collection of papers "Antibiotics," Moscow.

- 1959. La classification des actinomycetes par la methode de la variation experimentale. Ann. Inst. Pasteur. T.96. p. 434.

- 1960. On principles for classifying actinomycetes. Collection of papers "Biology of Individual Groups of antibiotic-producing actinomyces. Moscow.

. 1960. Taxonomic principles in the Actinomycetes.

Jour. Bact. 79(1):65.

- 1960. Rules for the classification of antibioticproducing actinomycetes. Jour. Bact. $\underline{79}(1): 75$. 
, and S.A. Egoro a. 1960. Recovery of pigmentation and antibiotic properties in Leucomutants-A. coelicolor with the aid of microbe metabolite. DAN SSSR.

, A.I. $K_{0}$ renyako, N.I. Nikitina, and G.K. Skryabin. 1951. Intra - and interspecies antagonism as a principle for identification and classification of species in microorganisms. DAN SSSR, $77(4): 725$.

Kriss, A.E. 1936. On pigments in actinomycetes. Mikrobiol. $\underline{5}(5)$.

, E.A. Rukina, and B.M. Isayev. 1945. Electron microscope study of actinomycete structure. Mikrobiol. 14:172.

Küster, E. 1953. Beitrage zur Genese und Morphologie der Streptomyces-spores. Atti Vi. Congr. Internatl. Microbiol. I. p.114.

Leshevalier, H.A. and A.S. Tihonienko. 1960. Effect of nutrition conditions on structure of surface of actinomycete spores. Mikrobiologia. 29(1).

Nikitina, N.I. 1957. Actinomycetes of Globisporinous Group. Thesis, Moscow.

Preobrazhenskaya, T.P., E.S. Kudrina, et al. 1960. On diagnostic significance of various indications in classification of representatives of Actinomyces genus. $29(3)$.

Pridham, T. and D. Gottlieb. 1948. The utilization of carbon compounds of some Actinomycetales as an aid for species determination. Jour. Bact. 56:107.

C. Hesseltine, and R.A. Benedict. 1958. A guide for the classification of streptomycetes according to selected groups. Appl. Microbiol. 6:52.

Tesic, Z.P. 1960. On rational classification of Actinomycetes. Mikrobiol. 29(4).

Waksman, S.J. 1959. The Actinomycetes. Vol.1. - 1959. Classification of Actinomycetes with special reference to Act. Mikrobiologia $\underline{28}(5)$.

Yamaguchi, T. and Y. Saburi. 1955. Studies on the antitrichomonal actinomycetes and their classification. I. Gener. Appl. Microbiol. (Japan) I $: 201$. 
Page 160

INTERNATIONAL BULLETIN

\section{EDITORIAL NOTE:}

The illustrations to accompany Dr. Krasil'nikov's article (this Bulletin pp. 133 to 159) have been delayed in the post. They will be published in a later issue. 